\title{
Privacy Preserving and Effective User Revocation on Dynamic Groups using CP-ABE Scheme
}

\author{
M. Sravani Kumari \\ M.Tech(CSE) \\ KHIT,Guntur, \\ India
}

\author{
K. Santhi \\ Assistant Professor(Dept.of \\ CSE) \\ KHIT, Guntur, \\ India.
}

\author{
B. Tarakeswara Rao, PhD \\ Professor(Dept.of CSE) \\ KHIT, Guntur \\ India
}

\begin{abstract}
Now a days the importance of cloud computing emerge with many sectors in order to reduce economical Cost and improve the revenue with improving cloud service usability like IaaS, SaaS and PaaS services. In this connection when group users outsource their data to the cloud among the dynamic group no assurance about user privacy, data integrity due to lack of data leakage and modifications among group users, such a case user authenticity, user data privacy and user revocation is to be a challenging issue with this system, thus to address all above issues we proposed a novel framework i.e. CP-ABE based secure public auditing for protecting data integrity and authenticity without compromising security and authenticity and also for effective user revocation on dynamic group members.
\end{abstract}

\section{Keywords}

CP-ABE Scheme, User Revocation, data integrity, Privacy.

\section{INTRODUCTION}

Persons will effortlessly cooperate as a cluster by imparting data to one another with data storage and sharing gave by the cloud. Once a client transfer shared data in the cloud, all clients in the cluster will don't just get to and change shared data, additionally share the most recent variant of the common data with whatever is left of the gathering. Despite the fact that cloud suppliers guarantee a more secure and trusted environment to the clients, because of the presence of equipment/programming disappointments and human blunders the trustworthiness of data in the cloud may in any case be traded off. The vast majority of the past works focus on examining the uprightness of individual data. Not quite the same as these works, a percentage of the late works focus on the most proficient method to save personality security from open verifiers while examining the respectability of shared data. Tragically, nothing unless there are other options techniques considers the productivity of client repudiation while evaluating the rightness of shared data in the cloud. With shared data, when a client did a few changes in a Blocklevel, she additionally needs to ascertain another mark for the changed square. Because of the changes from diverse clients, distinctive Block-levels are marked by distinctive clients. For security reasons, once a client leaves the cluster or gets into mischief, this client ought to be repudiated from the cluster. Therefore, this denied client should not have the capacity to get to and alter shared data; furthermore, the marks produced by this disavowed client aren't any more substantial to the cluster. In this way, however the substance of shared data isn't changed all through client denial, the obstructs that were prior marked by the repudiated client still must be constrained to be re-marked by partner degree existing client inside of the cluster. Subsequently, the trustworthiness of the entire data will at present be checked with the overall population keys of existing clients exclusively. Since shared data is outsourced to the cloud and clients not store it on local gadgets, a simple technique to re-register these marks all through client denial is to raise partner degree existing client to first exchange the Block-levels previously marked by the repudiated client, check the rightness of those squares, then re-sign these Blocklevels, and in the end exchange the new marks to the cloud. Be that as it may, this undemanding system could esteem the present client a colossal amount of correspondence and calculation assets by downloading and corroborative squares, and by precomputing and downloading marks, especially, once the amount of re-marked Block-levels is kind of enormous or the participation of the cluster is generally powerful. To make this matter much more terrible, existing clients could get to their data imparting administrations gave by the cloud to asset confined gadgets, similar to cell telephones, that any keeps existing clients from keeping up the accuracy of shared data speedily all through client disavowal Data sharing among group members shown as Venn Group member diagram

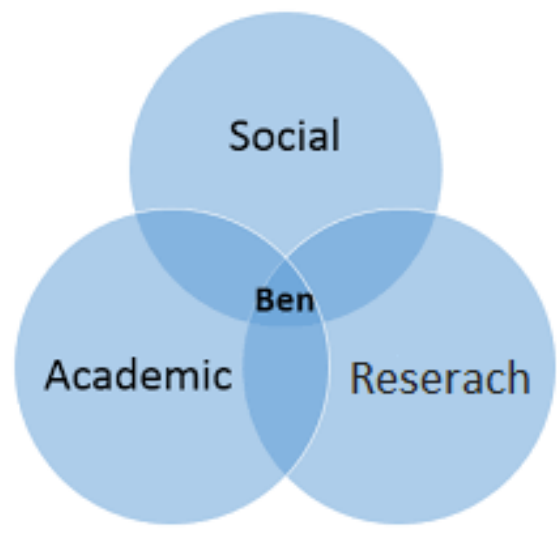

Fig 1. Venn Group members sharing

In this paper, we propose novel public auditing mechanism for the trustworthiness of shared data with productive client revocation in the cloud. In our system, by using the thought of intermediary re-signatures, when a client in the cluster is revoked, the cloud can resign the Block-levels, which were marked by the revoked client, with a re-signing key.

As a result, the strength of client revocation is regularly impressively enhanced, and calculation and correspondence resources of existing clients are frequently just spared. In the mean time, the cloud, that isn't inside of the same sure space with each client, is only ready to change over a signature of 
the revoked client into a signature of partner degree existing client on indistinguishable square, in any case, it can't sign supreme Block-levels in the interest of either the revoked client or partner degree existing client. By concocting a fresh out of the plastic new intermediary re-signature subject with decent

\section{LITERATURE SURVEY}

Boyang Wang, Baochun $\mathrm{Li}$ and Hui $\mathrm{Li}$ are the members of IEEE explore the concept of "Oruta: Privacy-Preserving Public Auditing for Shared data in the Cloud" in 2014. It shows that services of cloud provide not only data storage in commonplace, but also data sharing across multiple users. However, it remains an open challenge to audit the shared data by preserving identity privacy. This system proposed public auditing of shared data stored in cloud by using privacy preserving mechanism. In particular, this paper exploits the concept of group signature which computes the verification information required for integrity auditing of shared data. With this mechanism, the signer identity of each block in shared data remains private from a third party auditor (TPA) which can publicly verify shared data integrity without accessing entire data. In extend this mechanism support batch auditing. This mechanism is responsible for auditing multiple shared data in just single auditing task. The high level comparison between Oruta and its relevant existing systems are shown in following Table 1. This paper represent first attempt towards designing effective public auditing of shared data in the cloud storage by preserving privacy.

The work in this paper involves three parties: the cloud server, the third party auditor (TPA) and users. There are two types of users in a group: the original user and a number of group users. The original user and group users are both members of the group. Group members are allowed to access and modify shared data created by the original user based on access control polices. But this paper only shows that how to audit shared data integrity in a cloud with static group. It means groups of users already defined in cloud before shared data and membership of user is not changed during data sharing. The original user is responsible for deciding who is able to share its data before uploading data to the cloud. Another interesting problem is how to audit the integrity of shared data in the cloud with dynamic groups a new user can be added into the group and an existing group member can be revoked during data sharing while still preserving identity privacy.

\section{EXISTING SYSTEM}

In this presented system, a signature is attached with each Block-level in erudition, and, therefore, the data integrity relies on upon the correctness of the considerable number of signatures. One among the foremost imperative and standard choices of those instruments is to allow an open companion to with proficiency check information uprightness inside of the learning server while not downloading the entire learning, remarked as open inspecting. This open voucher may well be a customer who might really want to use data Server data for particular capacities or an Third party auditor (TPA) why should ready supply confirmation administrations on data uprightness to clients. The vast majority of the previous works represent considerable authority in inspecting the trustworthiness of non-open learning. Entirely unexpected from these works, numerous recent works have practical experience in an approach to preserve character protection from open verifiers once evaluating the uprightness of shared information. Unfortunately, none of the on top of components considers the power of client revocation once evaluating the correctness of shared information inside of the learning server [4]. A great deal of exceptionally, temperate clients revocations are regularly accomplished through an open revocation list while not change the individual keys of the remaining clients, and new clients will directly interpret documents hang on inside of the cloud before their cooperation. Moreover, the capacity overhead and, therefore, the coding calculation esteem are steady. So much on the whole, cases beat the present methodologies [5].

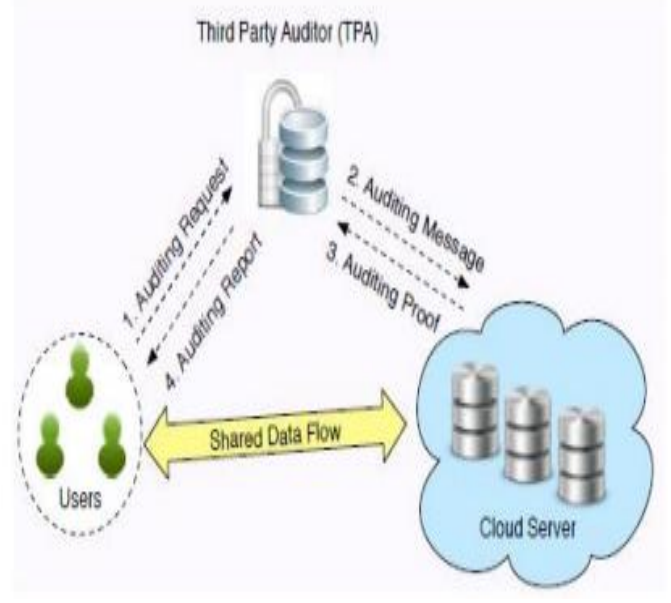

Fig 2.Presented System model

A. Drawbacks of Existing System: As a result, this revoked user should now not have the capacity to get to and change shared data; furthermore, the signatures produced by this revoked user are not any more substantial to the cluster. Therefore, however, the substance of shared data is not changed all through user revocation the hinders that are prior marked by the revoked user still must be constrained to be remarked by partner degree existing user inside of the cluster. The many of resigned Block-levels is slightly monster or the participation of the cluster is frequently alert. A simple system may esteem the prevailing user a huge amount of correspondence and calculation resources. Lows security Simple to locate the key to retrieve the information, Poor execution, High in memory use and time relevance process.

\section{PROPOSED SYSTEM}

In this paper, we consider the issue of building public authentication review for shared dynamic information with gathering user revocation. Our commitments are:

1) For figure content database, we explore on the secure and proficient shared information incorporate auditing for multiuser operation.

2) We mean an effective information auditing plan alongside new features, for example, $\mathrm{CP}-\mathrm{ABE}$ based secure uneven gathering key agreement and gathering signature.

3) The investigation results demonstrate that our plan is secure and productive as we give the security and effectiveness examination of our plan which will result in go down and information stockpiling in the cloud.

4) The approved copy check in the half and half cloud architecture is upheld by a few deduplication developments and this approved copy check conspire relatively acquires least overhead than ordinary operations. 


\section{PROPOSED WORK}

The anticipated ways to deal with Group Level Signature (GLS) a totally one of a kind public auditing instrument for the respectability of shared information with efficient user revocation inside of the $\mathrm{CP}-\mathrm{ABE}$ based for the most part information handling. Amid this component, by using the considered intermediary signatures, once a user in the cluster is revoked, the ABE-based for the most part information preparing is in a position to re-sign the obstructs that were marked by the revoked user, with a resigning key. As a result, the strength of user revocation may be significantly enhanced; calculation and correspondence resources of existing users may be basically spared. Inside of the anticipated instrument is ascendable, that shows it is not singularly ready to speedily bolster an outsized assortment of users to share learning and however conjointly ready to handle various auditing assignments in the meantime with cluster auditing [6]. Moreover, by taking advantages of Shamir Secret Sharing (SSS) might likewise augment our system into the multiintermediary model to weaken the possibility of the abuse on re-signing keys inside of the $\mathrm{ABE}$ on learning server based for the most part information preparing and enhances the responsibility of the complete component $[6,7]$. A. Focal points of Proposed Work: This component will extensively enhance the power of user revocation. The key composed agreement downside can be resolved by escrow-free key issue convention that is constructed misuse the secure two-party calculation between the key era focus and therefore the information putting away focus. Fine-grained user revocation per each trait may be finished as a substitute secret written work that exploits the particular characteristic cluster key appropriation on prime of the CP-ABE. High unevenness Enhanced execution better results Low in memory utilization

\section{SYSTEM ARCHITECTURE}

A system design or systems design is that the abstract model that defines the structure, behavior and additional views of a system. A design description could be a formal description and illustration of a system, organized in an exceedingly means that supports reasoning concerning the structures and behaviors of the system.

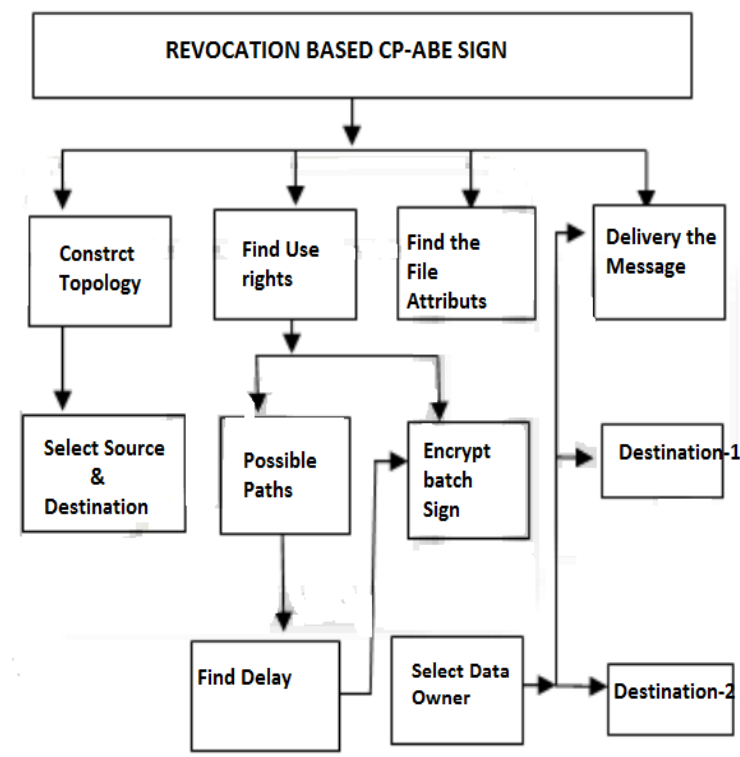

Fig 3. Revocation CP-Attribute Based Encryption on Group Level Processing
The system to explain the protection issue on shared information, a novel security safeguarding open evaluating component. Cloud Administration suppliers transferring the data to cloud user from the cloud server. At present, the TPA must be constrained to check the honesty of exchanged data. The system will be similar to this; The TPA can gather the get data and send data to check. In the event that every data same, then there's no infringement inside of the data uprightness. Much this is regularly tough for the enormous data. Furthermore, TPA also an outer substance yet again in the event that we tend to offer the full arrangement of data afresh information trustworthiness inquiry can ascend in TPA wrap up. For the numerous cloud and various users, we'd like different reviewing known as clump inspecting. We'd like to actualize the new procedure with Homomorphic authenticator and, in this manner, the added substance blend signature technique. To build people, in general, inspecting system, we will augment the ring mark plan. The idea of ring signatures is initially proposed by Rivest et al. in 2001. With ring signatures, a verifier is persuaded that a mark is processed utilizing one of group part's private keys; however, the verifier is not ready to figure out which one. This property can be utilized to protect the personality of the underwriter from a verifier. The ring mark plan presented by Boneh et al. (Alluded to as BGLS in this paper) is developed on bilinear maps [5] . Chiefly four segments are incorporated into this system. They are Customer, Owner, Cloud server, and Outsider Reviewer. In any case, here Outsider reviewer will convey vital part in this system. The owner gives the information's to the shopper through the Cloud server. The respectability of the data inside of the Cloud server is in the accentuation mark. The owner will check the uprightness of auditing so as to learn in Cloud server. However having examining inside of the owner is valued viable and it results in a cerebral pain to the owner. The answer is to have a TPA to check the trustworthiness of information inside of the Cloud server. The Outsider Examiner could be an impartial element to the Cloud Server furthermore the Owner. In the interest of the owner, the TPA can confirm the owner's information stockpiling and security system. TPA should be a trusty element. However believing a third gathering isn't sensible. To ensure the security of the information, the data substance isn't out there to the Outsider Reviewer. The TPA checks the encoded learning all together that the security of the data is guaranteed. This will be done abuse Homomorphic confirmation. The data is created misuse Homomorphic validation. The TPA debate the Cloud server for the evidence of learning uprightness. The Cloud server gives the verification that is checked against the owner's data. The protection safeguarding open examining utilizing signatures incorporate three calculations as already said here: KeyGen: Every user in the group produces their public key and private key. Ring Sign: User in the group identifies with sign a square with her private key and all group memberse ${ }^{\text {ee }}$ open keys. Ring confirm: The verifier can be utilized to test if the given square is marked by the group part here ring confirmation applies CP-ABE Scheme to give the access rights .

\section{CONCLUSION}

In this paper we proposed a primitive solution for privacy preserving for outsourced cloud data among group users and also provide details on effective user revocation on dynamic group hence to enhance the efficiency of verifying multiple auditing tasks using $\mathrm{CP}-\mathrm{ABE}$ Scheme at Verifier level, it further extends this mechanism to guide batch auditing. This 
mechanism is provide reduced signature storage and also supports dynamic operations on shared data.

\section{REFERENCES}

[1] S. Grance, "Draft NIST working definition of Data Server computing".

[2] C. Wang, Q. Wang, K. Ren, and W. Lou, "Towards Secure and Dependable Storage Services in Cloud Computing," IEEE Transactions on Services Computing, vol. 5, no. 2, pp. 220-232, 2011.

[3] Y. Zhu, G.-J. Ahn, H. Hu, S. S. Yau, H. G. An, and S. Chen, "Dynamic Audit Services for Outsourced Storage in Clouds," IEEE Transactions on Services Computing, accepted.

[4] S. Marium, Q. Nazir, A. Ahmed, S. Ahthasham and Aamir M. Mirza, "Implementation of EAP with RSA for Enhancing The Security of Cloud Computing", International Journal of Basic and Applied Science, vol 1, no. 3, pp. 177-183, 2012.

[5] Balkrishnan. S, Saranya. G, Shobana. S and Karthikeyan.S, "Introducing Effective Third Party Auditing (TPA) for Data Storage Security in Cloud", International Journal of computer science and Technology, vol. 2, no. 2, ISSN 2229-4333 (Print) | ISSN: 0976- 8491(Online), June 2012.
[6] K. Kiran Kumar, K. Padmaja, P. Radha Krishna, "Automatic Protocol Blocker for Privacy-Preserving Public Auditing in Cloud Computing", International Journal of Computer science and Technology, vol. 3 pp, ISSN. 0976-8491(Online), pp. 936-940, ISSN: 22294333 (Print), March 2012.

[7] Jachak K. B., Korde S. K., Ghorpade P. P. and Gagare G.J., "Homomorphic authentication with Random Masking Technique Ensuring Privacy \& Security in Cloud Computing", Bioinfo Security Informatics, vol. 2, no. 2, pp. 49-52, ISSN. 2249-9423, 12 April 2012.

[8] J. Yuan and S. Yu, "Proofs of Retrievability with Public Verifiability and Constant Communication Cost in Cloud," in Proceedings of ACM ASIACCS-SCC'13, 2013.

[9] H. Shacham and B. Waters, "Compact Proofs of Retrievability,"in the Proceedings of ASIACRYPT 2008. Springer Verlag,2008,pp.90-107.

[10] G. Ateniese, R. Burns, R. Curtmola, J. Herring, L. Kissner, Z. Peterson, and D. Song, "Provable Data Possession at Untrusted Stores,"in the Proceedings of ACM CCS 2007, 2007, pp. 598-610. 